\title{
Introduction to the Internet of Things: Providing Services Using Smart Devices, Wearables, and Quantified Self Minitrack
}

\author{
Tayfun Keskin \\ The University of Texas Rio Grande Valley \\ keskin@utexas.edu
}

This minitrack addresses Internet-of-Things (IoT) enabled issues that people, teams, organizations, and institutions face as they seek to utilize connected products and services. These products and services include smart devices, wearables, or other processes that facilitate quantification. The IoT allows connectivity of billions of devices that can sense, store data, and provide analytical services to users. IoT-enabled ecosystems enhance teamwork and project management disciplines through creating value for team members. In addition, IoT enhances games (and other interactive digital services) because it connects gamers, which enhances their utility. Gamification of other processes could also use IoT. For example, teams can create intrinsic value by measuring and sharing team member data. Finally, we predict that autonomous mobile devices will have the ability to move and actuate on their surroundings in an effort to provide services to users who may or may not be in physical proximity of the devices. These scenarios create business opportunities for organizations seeking to provide services to these users.

However, these opportunities create numerous questions that have not been adequately researched including applicable business models for these services, how value is co-created in these service settings, the role of network externalities, security and privacy issues, data analysis issues and big data techniques, and how mobile devices will make contact with other devices and form business relationships. In addition, very few studies have been conducted on using the Internet of Things to provide such services.

The three papers accepted for the minitrack investigate these issues.

The first paper, titled "The Internet of Things, Teamwork, and Process Improvement" by Ziye (Randy) Wang and Deanna Kennedy demonstrates how IoT enhance teams and processes. The Internet of Things (IoT) has made inroads into many aspects of our lives. The application of IoT has enhanced our homes, such as with smart thermostats, as well as our workplace, with RFID sensors for tracking and replenishing resources. Yet, there is limited research about the way human processes, and in particular, team processes are changed by IoT objects. Herein, they distinguish IoT objects based on

URI: http://hdl.handle.net/10125/50050

ISBN: 978-0-9981331-1-9

(CC BY-NC-ND 4.0) functionality; with activity-aware objects tracking and recording data, policy-aware objects that can take limited actions according to given rules (e.g., sending alerts), and process-aware objects that can make dynamic decisions or augment employee decision making. They draw on the team process framework to clarify the type of task-oriented activities team members engage in overtime as they collectively complete work projects. They also connect different types of IoT objects to relevant team processes and propose that the synergies may enhance team efficiencies and effectiveness. Finally, they provide a real-world scenario about air conditioning service that illustrates how different IoT objects may be introduced to affect the team processes.

The second paper, titled "Pricing of Games as a Service: An Analytical Model for Interactive Digital Services with Hedonistic Properties" by Tayfun Keskin investigates pricing strategies of games in a connected and multi-sided setup. His study explores optimal pricing strategies in games and other interactive digital goods under incomplete information, when bundling is an option. Drawing from research on the pricing of information goods, we propose a pattern of optimal pricing strategies in which hedonic characteristics affect the utility of interactive digital goods and services. This is a new approach to games, to treat them as a service to determine pricing strategies. Findings reveal that there is an optimal pricing solution for firms in the gaming industry. This finding holds both in bundling and nonbundling cases. Utilizing analytical modeling methodology, he proposes pricing-inspired business strategies to the firms operating in the digital gaming industry. His findings could also be applied to other hedonic interactive digital goods and services. Overall, this study contributes to the existing pricing theories in digital services and information goods.

The third paper, titled "Pricing of Virtual Goods and Designing Game Challenge Level for Free-to-Play Mobile Games in the Presence of Copycat Competitors" by Ismail Civelek, Yipeng Liu, and Sean Marston is another study focusing on mobile games. In-game purchases, virtual currency, content design for heterogeneous consumers and strong competition are key challenges for mobile game providers. This study addresses determination of optimal game design strategies for

Page 1329

\section{HîCSS}


game providers in the presence of heterogeneous players and copycat competitors. Moreover, this paper incorporates pricing of virtual goods/currency into the Free-to-Play (F2P) mobile game design via a duopoly model and characterizes the optimal strategies for game providers in terms of pricing of virtual goods/currency and the game challenge level.

We hope you enjoy the papers and their presentation at the conference. We thank the authors for submitting their work to make this another engaging minitrack. We also thank the reviewers for their valuable feedback. 